Discrete Comput Geom 34:497-506 (2005)

DOI: $10.1007 / \mathrm{s} 00454-005-1177-\mathrm{z}$

\title{
On the Chromatic Number of the Visibility Graph of a Set of Points in the Plane*
}

\author{
Jan Kára, ${ }^{1}$ Attila Pór, ${ }^{2}$ and David R. Wood ${ }^{3}$ \\ ${ }^{1}$ Department of Applied Mathematics, Charles University, \\ 11800 Prague 1, Czech Republic \\ kara@kam.mff.cuni.cz \\ ${ }^{2}$ Department of Mathematics, Case Western University, \\ Cleveland, $\mathrm{OH} 44106$, USA \\ attila.por@case.edu \\ ${ }^{3}$ Departament de Matemàtica Aplicada II, \\ Universitat Politècnica de Catalunya, \\ E-08034 Barcelona, Spain \\ wood@cs.mcgill.ca
}

\begin{abstract}
The visibility graph $\mathcal{V}(P)$ of a point set $P \subseteq \mathbb{R}^{2}$ has vertex set $P$, such that two points $v, w \in P$ are adjacent whenever there is no other point in $P$ on the line segment between $v$ and $w$. We study the chromatic number of $\mathcal{V}(P)$. We characterise the 2 - and 3 -chromatic visibility graphs. It is an open problem whether the chromatic number of a visibility graph is bounded by its clique number. Our main result is a super-polynomial lower bound on the chromatic number (in terms of the clique number).
\end{abstract}

\section{Introduction}

Let $P \subseteq \mathbb{R}^{2}$ be a set of points in the plane. Let $\overline{v w}$ denote the closed line segment between points $v \in \mathbb{R}^{2}$ and $w \in \mathbb{R}^{2}$. Two distinct points $v, w \in P$ are visible with respect to $P$ if $P \cap \overline{v w}=\{v, w\}$. The visibility graph $\mathcal{V}(P)$ of $P$ has vertex set $P$, where two distinct points $v, w \in P$ are adjacent if and only if they are visible with respect to $P$.

* The research of A. Pór and D. Wood was completed in the Department of Applied Mathematics and the Institute for Theoretical Computer Science at Charles University, Prague, Czech Republic. Supported by project LN00A056 of the Ministry of Education of the Czech Republic, and by the European Union Research Training Network COMBSTRU (Combinatorial Structure of Intractable Problems). The research of D. Wood is supported by the Government of Spain Grant MEC SB2003-0270. 


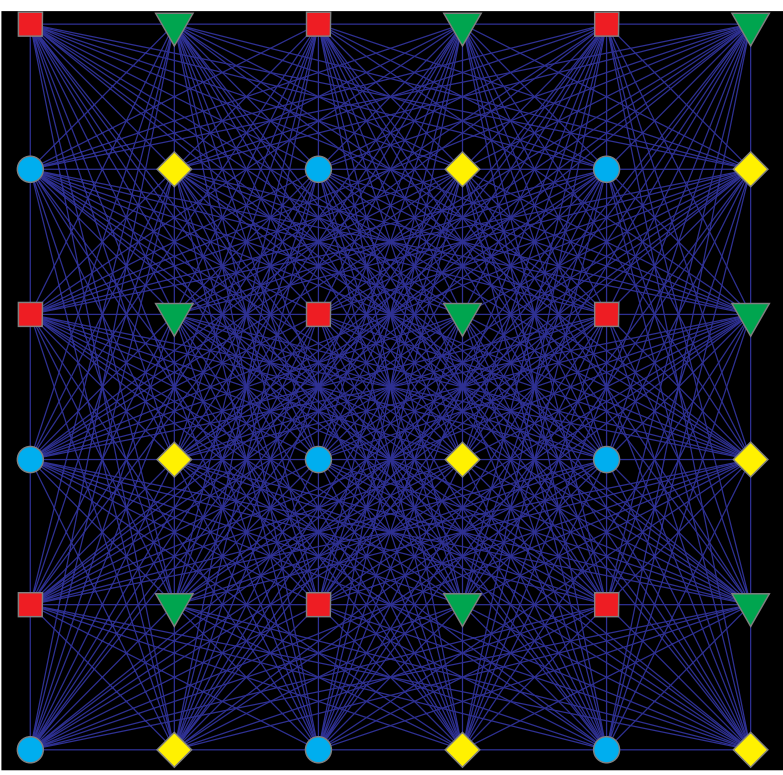

Fig. 1. 4-Colouring of the visibility graph of the integer lattice.

A $k$-colouring of a graph $G=(V, E)$ is a function $f: V \rightarrow C$ for some set $C$ of $k$ colours, such that $f(v) \neq f(w)$ for every edge $v w \in E$. We say $G$ is $k$-colourable. The chromatic number $\chi(G)$ is the minimum $k$ such that $G$ is $k$-colourable. The clique number $\omega(G)$ is the maximum $k$ such that $G$ has a $k$-clique.

This paper studies the chromatic number of visibility graphs. We begin with an interesting example.

Proposition 1. Let $P=\{(x, y): x, y \in \mathbb{Z}\}$ be the integer lattice. Then $\chi(\mathcal{V}(P))=4$.

Proof. Let $f((x, y))=(x \bmod 2, y \bmod 2)$ for all $(x, y) \in P$. For any two points $\left(x_{1}, y_{1}\right)$ and $\left(x_{2}, y_{2}\right)$ in $P$ for which $f\left(\left(x_{1}, y_{1}\right)\right)=f\left(\left(x_{2}, y_{2}\right)\right)$, both $\left|x_{1}-x_{2}\right|$ and $\left|y_{1}-y_{2}\right|$ are even. Thus the midpoint of the segment $\overline{\left(x_{1}, y_{1}\right)\left(x_{2}, y_{2}\right)}$ is in $P$, and $\left(x_{1}, y_{1}\right)$ and $\left(x_{2}, y_{2}\right)$ are not visible. Hence $f$ is a 4-colouring of $\mathcal{V}(P)$, as illustrated in Fig. 1. There is no 3 -colouring since $\{(0,0),(1,0),(1,1),(0,1)\}$ is a 4-clique. Therefore $\chi(\mathcal{V}(P))=4$.

While the visibility graph of the integer lattice has a quadratic number of edges, Proposition 1 proves that it has a small chromatic number. Also note that Proposition 1 generalises to prove that the visibility graph of the $d$-dimensional ${ }^{1}$ integer lattice is $2^{d}$. In this case the chromatic number and the clique number coincide. ${ }^{2}$ Whether there is a similar relationship for all visibility graphs is a fundamental open problem.

\footnotetext{
${ }^{1}$ Note that the visibility graph of a set of points in $\mathbb{R}^{d}$, by a suitable projection, is also a visibility graph of some set of points in $\mathbb{R}^{2}$.

2 The visibility graph of the integer lattice is not perfect. For example, $((2,5)(1,3)(5,8)(8,3)(5,1))$ is an induced 5-cycle.
} 
Conjecture 1. Visibility graphs are $\chi$-bounded. That is, is there a function $f$ such that $\chi(\mathcal{V}(P)) \leq f(\omega(\mathcal{V}(P)))$ for every finite point set $P$ ?

In Section 2 we make some observations about visibility graphs, and give an elementary bound on their chromatic number. In Section 3 we prove that in Conjecture 1, we can take $f(2)=2$ and $f(3)=3$. In fact we characterise the finite point sets whose visibility graph has chromatic number 2 or 3 . The main result of this paper, presented in Section 4, is a super-polynomial lower bound on the chromatic number in terms of the clique number, for a certain family of visibility graphs. We conclude in Section 5 with a discussion of visibility graphs with $\omega(\mathcal{V}(P))=4$.

Note that visibility graphs of polygons are well studied (see [1] for example); even here, it is an open problem whether the chromatic number is bounded by the clique number. The main open problem that has been studied here is whether visibility graphs of polygons can be recognised in polynomial time (see [3] for example). This question is also of interest for general visibility graphs of point sets. See [6], [7], and [9] for results and open problems regarding the $\chi$-boundedness of other graph families that arise in a geometric context.

\section{Observations}

Thee following is a fundamental observation regarding visibility graphs.

Proposition 2. For every finite point set $P \subset \mathbb{R}^{2}$, the diameter of the visibility graph $\mathcal{V}(P)$ is

$$
\begin{cases}1 & \text { if } P \text { is in general position }, \\ |P|-1 & \text { if } P \text { is collinear } \\ 2 & \text { otherwise. }\end{cases}
$$

Proof. The diameter is 1 if and only if $\mathcal{V}(P)$ is complete, which occurs if and only if $P$ is in general position. If $P$ is collinear, then $\mathcal{V}(P)$ is a path, which has diameter $|P|-1$. Thus it suffices to prove that if $P$ is not in general position and not collinear, then the diameter of $\mathcal{V}(P)$ is 2 . Consider two non-visible points $v, w \in P$. Two such points exist, since $P$ is not in general position. Let $L$ be the line containing $v$ and $w$. Let $x$ be a point in $P$ not on $L$, such that the perpendicular distance from $x$ to $L$ is minimised. There is such a point $x$ as $P$ is finite, and not all the points in $P$ are collinear. Then $v$ and $x$ are visible and $w$ and $x$ are visible, as otherwise there is a point in $P$ closer to $L$ than $x$. Thus the distance from $v$ to $w$ in $\mathcal{V}(P)$ is 2 . Hence the diameter of $\mathcal{V}(P)$ is 2 .

Here is one way to colour $\mathcal{V}(P)$.

Proposition 3. If a point set $P \subseteq \mathbb{R}^{2}$ can be covered by $k$ lines, then $\chi(\mathcal{V}(P)) \leq 2 k$.

Proof. Associate each point $v \in P$ with one of the $k$ lines that contain $v$. The subgraph of $\mathcal{V}(P)$ induced by the set of points assigned to any one line is a collection of disjoint 
paths, and is thus 2-colourable. Using a different pair of colours for each line we obtain a $2 k$-colouring of $\mathcal{V}(P)$.

Corollary 1. For every point set $P \subseteq \mathbb{R}^{2}, \chi(\mathcal{V}(P))$ is at most twice the minimum degree of $\mathcal{V}(P)$.

Proof. The result follows from Proposition 3, since $P$ clearly can be covered by $\operatorname{deg}(v)$ lines for any point $v \in P$.

\section{The 2- and 3-Chromatic Visibility Graphs}

In what follows we characterise the finite point sets whose visibility graph has chromatic number 2 or 3.

Theorem 1. Let $P$ be a finite point set. Then the following are equivalent:

(a) $\chi(\mathcal{V}(P)) \leq 2$,

(b) all the points in $P$ are collinear,

(c) $\mathcal{V}(P)$ has no $K_{3}$ subgraph.

Proof. That (a) implies (c) is immediate. If all the points in $P$ are collinear, then $\mathcal{V}(P)$ is a path, which is obviously 2 -colourable. Thus (b) implies (a). It remains to prove that (c) implies (b). Suppose that not all the points in $P$ are collinear. Let $\{u, v, w\}$ be a set of three non-collinear points in $P$ such that the triangle $u v w$ has minimum area. If there is a distinct point $x \in P \cap \overline{u v}$, then $\{x, v, w\}$ are non-collinear and the triangle $x v w$ has less area than $u v w$, which is a contradiction. Thus $u$ and $v$ are visible. Similarly $u$ and $w$ are visible, and $v$ and $w$ are visible. Hence $\{u, v, w\}$ induce $K_{3}$ in $\mathcal{V}(P)$.

Before characterising the 3-colourable visibility graphs, consider when $\mathcal{V}(P)$ is planar. In $\mathcal{V}(P)$ there is a line segment between every pair of vertices (which may be comprised of many edges). Dujmović et al. [5] characterised those planar graphs in which there is a line segment between every pair of vertices, as illustrated in Fig. 2.

Lemma 1 [5]. Let $P$ be a point set. Then $\mathcal{V}(P)$ is planar if and only if at least one of the following conditions hold:

(a) all the points in $P$ are collinear,

(b) all the points in $P$, except for one, are collinear,

(c) all the points in $P$ are collinear, except for two non-visible points,

(d) all the points in $P$ are collinear, except for two points $v, w \in P$, such that the line segment $\overline{v w}$ does not intersect the line segment that contains $P \backslash\{v, w\}$,

(e) $\mathcal{V}(P)$ is an affine transformation of the octahedron drawn in Fig. 2(e). 
(e)

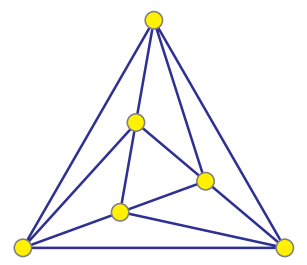

(b)

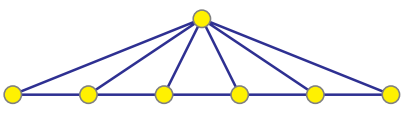

(a)

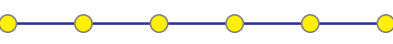

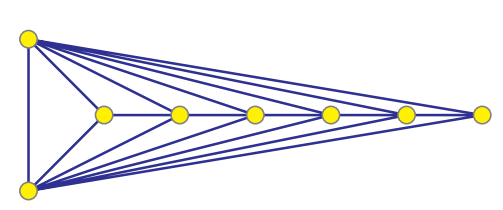

(d)

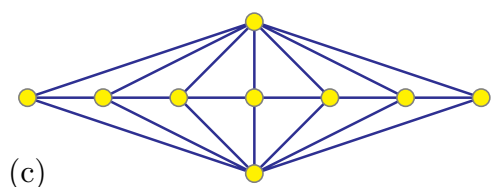

Fig. 2. The planar visibility graphs.

Theorem 2. Let $P$ be a finite point set. Then the following are equivalent:

(i) $\chi(\mathcal{V}(P)) \leq 3$,

(ii) P satisfies conditions (a), (b), (c) or (e) in Lemma 1,

(iii) $\mathcal{V}(P)$ has no $K_{4}$ subgraph.

Proof. That (i) implies (iii) is immediate. It is easy to construct a 3-colouring of a visibility graph that satisfies conditions (a)-(c) or (e) in Lemma 1. Thus (ii) implies (i). It remains to prove that (iii) implies (ii). Suppose that $\mathcal{V}(P)$ has no $K_{4}$ subgraph. Develin et al. [4] proved that a visibility graph is planar or contains $K_{4}$. (This result applies to a broad range of visibility graphs that includes visibility graphs of point sets.) Thus $\mathcal{V}(P)$ is planar. Lemma 1 describes all the planar visibility graphs. Of these only those satisfying condition (d) contain $K_{4}$.

\section{A Lower Bound}

In this section we prove the following super-polynomial lower bound on the chromatic number of a visibility graph.

Theorem 3. There are constants $c_{1}, c_{2}, c_{3}, c_{4}>0$ and an infinite sequence of visibility graphs $G_{0}, G_{1}, G_{2}, \ldots$, such that $\omega\left(G_{i}\right) \rightarrow \infty$ and

$$
\chi\left(G_{i}\right) \geq\left(c_{1} \log \omega\left(G_{i}\right)\right)^{c_{2} \log \omega\left(G_{i}\right)}=\left(c_{3} \omega\left(G_{i}\right)\right)^{c_{4}+\log \log \omega\left(G_{i}\right)} .
$$

Before proving Theorem 3 we recall two definitions from the literature. Let $G$ and $H$ be graphs. The lexicographic product of $G$ by $H$, denoted by $H[G]$, is the graph with vertex set $V(G) \times V(H)$, where $\{v a, w b\}$ is an edge if and only if $a b \in E(H)$, or $a=b$ and $v w \in E(G)$. The fractional chromatic number $\chi_{\mathrm{f}}(G)$ of a graph $G$ is the infimum of all fractions $a / b$ such that, to each vertex of $G$, one can assign a $b$-element subset of $\{1,2, \ldots, a\}$ in such a way that adjacent vertices are assigned disjoint subsets. Obviously 
$\chi_{\mathrm{f}}(G) \leq \chi(G)$. Scheinerman and Ullman [11] proved the following important property about the fractional chromatic number of the lexicographic product.

Lemma 2 [11]. For all graphs $G$ and $H, \chi_{\mathrm{f}}(H[G])=\chi_{\mathrm{f}}(H) \cdot \chi_{\mathrm{f}}(G)$.

Lemma 3. For every visibility graph $G$ and for every finite graph $H$, there is a visibility graph $X$ such that $\omega(H) \cdot \omega(G) \leq \omega(X) \leq \omega(H) \cdot \omega(G)+2|V(H)|$, and $\chi_{\mathrm{f}}(X) \geq$ $\chi_{\mathrm{f}}(H) \cdot \chi_{\mathrm{f}}(G)$.

Proof. Suppose $V(H)=\{1,2, \ldots, n\}$. Let $\left\{D_{1}, D_{2}, \ldots, D_{n}\right\}$ be a set of closed unit discs in the plane, whose centres are positioned on the vertices of a sufficiently large regular $n$-gon. For all $1 \leq i<j \leq n$, we say a segment with endpoints in $D_{i}$ and $D_{j}$ is an $i j$-segment. Here "sufficiently large" means that for each disc $D_{i}$ :

(1) the only disc that an $i j$-segment intersects is $D_{i}$ and $D_{j}$,

(2) there is a line $L_{i}$ such that every $i j$-segment crosses $L_{i}$,

(3) whenever an $i j$-segment crosses an $i k$-segment $(j \neq k)$, the crossing point is on the side of $L_{i}$ that contains $D_{i}$.

Scale $G$ so that its convex hull is enclosed in a unit disc and no vertex is at the centre of the disc. Let $\left\{G_{1}, G_{2}, \ldots, G_{n}\right\}$ be copies of $G$, one associated with each vertex of $H$. Place each $G_{i}$ in the disc $D_{i}$, rotated so that if three points in $\bigcup_{i} V\left(G_{i}\right)$ are collinear, then they are in a single $G_{i}$. This can be achieved by rotating each $G_{i}$ in turn. At each step, there are only finitely many forbidden rotation angles.

Let $X_{0}$ be the visibility graph defined by the point set $\bigcup_{i} V\left(G_{i}\right)$. By property (1) and the choice of orientations, every point in $G_{i}$ is visible with every point in $G_{j}$ for all $i \neq j$. Visibility within each $G_{i}$ is preserved by scaling and rotating. Thus $X_{0}=K_{n}[G]$.

We now introduce blocker points to our set, so that the subgraph of the visibility graph induced by $\bigcup_{i} V\left(G_{i}\right)$ is $H[G]$. For every non-edge $i j$ of $H$ (that is, an edge of $\bar{H}$ ), and for all vertices $p \in V\left(G_{i}\right)$ and $q \in V\left(G_{j}\right)$, add one blocker point at the intersection of the segment $p q$ and the line $L_{i}$, and add another blocker point at the intersection of the segment $p q$ and the line $L_{j}$. If two blocker points coincide, then just use one point. This construction is illustrated in Fig. 3.

Let $X$ be the visibility graph of the point set obtained. By property (3) above, for every edge $i j \in E(H)$, every vertex in $G_{i}$ is visible with every vertex in $G_{j}$. Thus the subgraph of $X$ induced by $\bigcup_{i} V\left(G_{i}\right)$ is $H[G]$.

Obviously $\omega(H[G])=\omega(H) \cdot \omega(G)$. The blocker vertices on each line $L_{i}$ can add at most two vertices to a maximum clique. Thus $\omega(H) \cdot \omega(G) \leq \omega(X) \leq \omega(H)$. $\omega(G)+2|V(H)|$, as claimed. By Lemma 2 and since $H[G]$ is an induced subgraph of $X, \chi_{\mathrm{f}}(X) \geq \chi_{\mathrm{f}}(H[G])=\chi_{\mathrm{f}}(H) \cdot \chi_{\mathrm{f}}(G)$, as claimed.

The following result of Larsen et al. [8] is based on the famous construction of Mycielski [10], which we include for completeness.

Lemma 4 [8]. For all $k \geq 0$, there is a triangle-free graph $M_{k}$ on $3 \cdot 2^{k}-1$ vertices such that $\chi_{\mathrm{f}}\left(M_{k}\right) \geq \sqrt{2 k}$. 


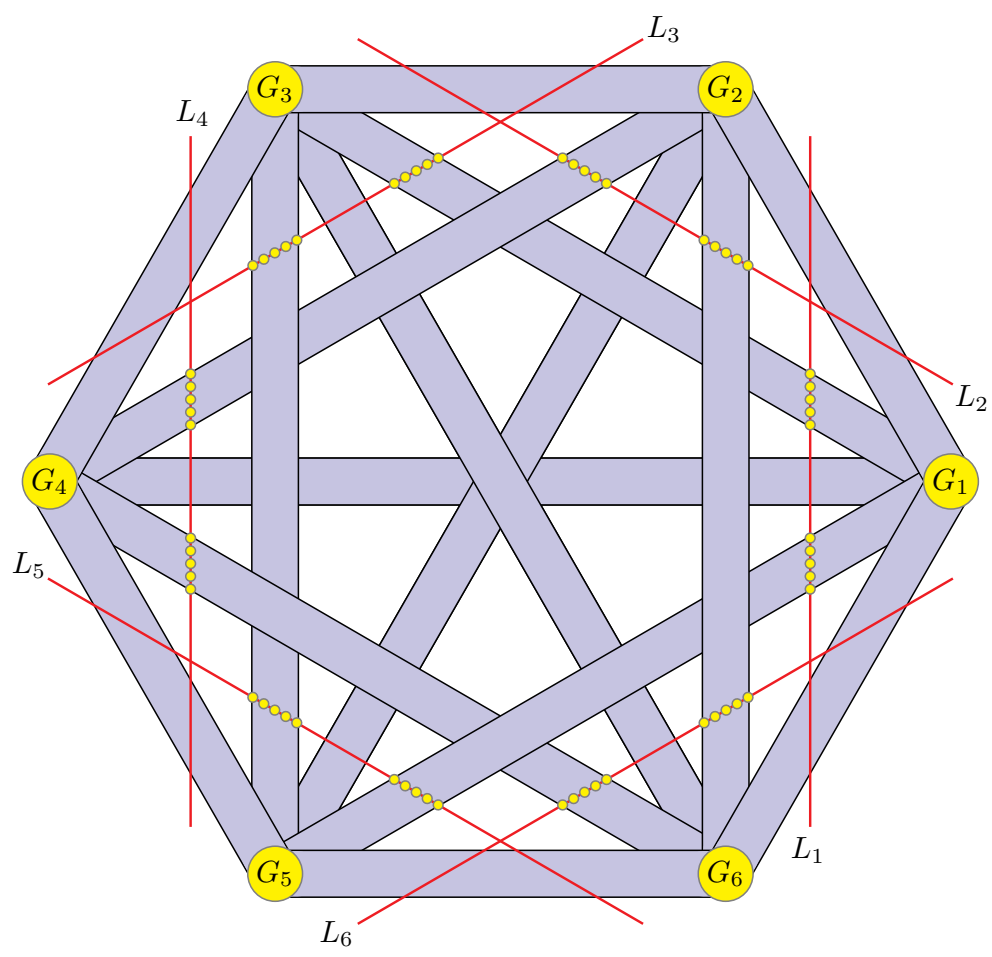

Fig. 3. Construction of $X$ from a visibility graph $G$ with $H=K_{3,3}$.

Proof of Construction. Let $M_{0}=K_{2}$. Construct $M_{k+1}$ from $M_{k}$ as follows. Suppose $V\left(M_{k}\right)=\left\{v_{i}: 1 \leq i \leq n_{k}\right\}$. Let $V\left(M_{k+1}\right)=\left\{x_{i}, y_{i}: 1 \leq i \leq n_{k}\right\} \cup\{z\}$. Let $E\left(M_{k+1}\right)=\left\{x_{i} x_{j}: v_{i} v_{j} \in E\left(M_{k}\right)\right\} \cup\left\{x_{i} y_{j}: v_{i} v_{j} \in E\left(M_{k}\right)\right\} \cup\left\{y_{i} z: 1 \leq i \leq n_{k}\right\}$. Note that $M_{k+1}$ has $n_{k+1}=2 n_{k}+1$ vertices. Since $n_{0}=2$, it follows that $n_{k}=3 \cdot 2^{k}-1$. Mycielski [10] proved that $M_{k}$ is triangle-free and $\chi\left(M_{k}\right)=k+2$. Larsen et al. [8] proved that $\chi_{\mathrm{f}}\left(M_{k+1}\right)=\chi_{\mathrm{f}}\left(M_{k}\right)+1 / \chi_{\mathrm{f}}\left(M_{k}\right)$. It follows that $\chi_{\mathrm{f}}\left(M_{k}\right) \geq \sqrt{2 k}$ (and this is asymptotically tight; see [8] and [11]).

Proof of Theorem 3. In what follows we make little effort to optimise the constants $c_{1}$ and $c_{2}$. Let $G_{0}=K_{1}$. For all $i \geq 0$, apply Lemma 3 to obtain a visibility graph $G_{i+1}(=X)$ from $G_{i}(=G)$, where $H$ is the Mycielski graph $M_{k(i)}$, chosen so that

$$
3 \omega\left(G_{i}\right) \leq\left|V\left(M_{k(i)}\right)\right|=3 \cdot 2^{k(i)}-1 \leq 6 \omega\left(G_{i}\right) .
$$

By Lemma 3 and since $M_{k(i)}$ is triangle-free,

$$
2 \omega\left(G_{i}\right) \leq \omega\left(G_{i+1}\right) \leq 2 \omega\left(G_{i}\right)+2\left|V\left(M_{k(i)}\right)\right| \leq 14 \omega\left(G_{i}\right),
$$

where the last inequality follows from (1). Since $\omega\left(G_{0}\right)=1$,

$$
2^{i} \leq \omega\left(G_{i}\right) \leq 14^{i} .
$$


We now prove a lower bound on the chromatic number of $G_{i+1}$. By Lemmata 3 and 4 ,

$$
\chi_{\mathrm{f}}\left(G_{i+1}\right) \geq \chi_{\mathrm{f}}\left(M_{k(i)}\right) \cdot \chi_{\mathrm{f}}\left(G_{i}\right) \geq \sqrt{2 k(i)} \chi_{\mathrm{f}}\left(G_{i}\right) .
$$

By (1) and the lower bound in (2), $k(i)>\log _{2} \omega\left(G_{i}\right) \geq i$. Hence,

$$
\chi_{\mathrm{f}}\left(G_{i+1}\right)>\sqrt{2 i} \chi_{\mathrm{f}}\left(G_{i}\right) .
$$

Since $\chi_{\mathrm{f}}\left(G_{0}\right)=1$,

$$
\chi_{\mathrm{f}}\left(G_{i}\right) \geq \sqrt{2^{i} i !}
$$

By Stirling's formula,

$$
\chi_{\mathrm{f}}\left(G_{i}\right) \geq \sqrt{(2 i / e)^{i}}
$$

By the upper bound in (2), $i \geq \log _{14} \omega\left(G_{i}\right)$. Hence

$$
\chi_{\mathrm{f}}\left(G_{i}\right) \geq\left(\frac{2}{\boldsymbol{e}} \log _{14} \omega\left(G_{i}\right)\right)^{(1 / 2) \log _{14} \omega\left(G_{i}\right)} .
$$

Obviously $\chi\left(G_{i}\right) \geq \chi_{\mathrm{f}}\left(G_{i}\right)$. Thus for an appropriate choice of constants $c_{1}, c_{2}>0$,

$$
\chi\left(G_{i}\right) \geq \chi_{\mathrm{f}}\left(G_{i}\right) \geq\left(c_{1} \log \omega\left(G_{i}\right)\right)^{c_{2} \log \omega\left(G_{i}\right)},
$$

as claimed.

\section{Future Directions}

We have proved Conjecture 1 for visibility graphs with $\omega(\mathcal{V}(P)) \leq 3$. The next interesting case is $\omega(\mathcal{V}(P))=4$. Figure 4 shows a visibility graph with $\omega(\mathcal{V}(P))=4$, for which it is easily seen that $\chi(\mathcal{V}(P))=5$. It is an open problem whether every visibility graph with $\omega(\mathcal{V}(P)) \leq 4$ has $\chi(\mathcal{V}(P)) \leq 5$.

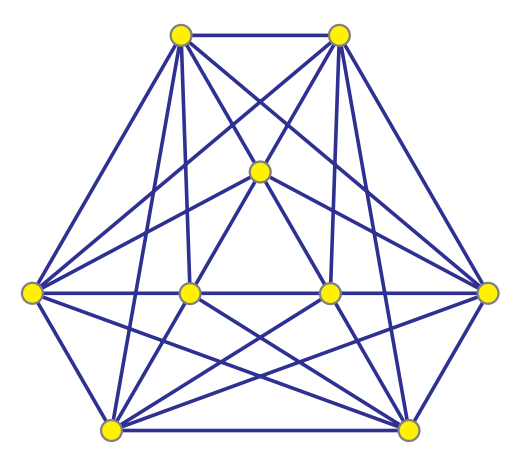

Fig. 4. A 5-chromatic visibility graph with maximum clique size 4 . 


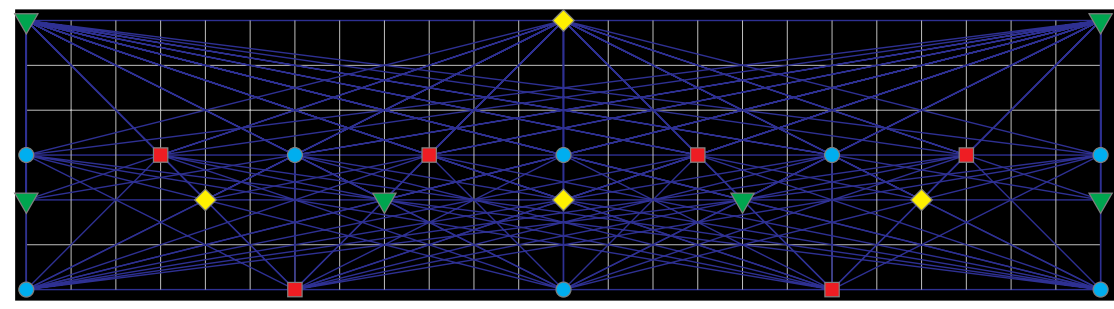

Fig. 5. An interesting example of a $K_{5}$-free visibility graph.

The diversity of point sets that satisfy $\omega(\mathcal{V}(P))=4$ is illustrated by the example shown in Fig. 5. Let $P=\{(12 i, 6),(3 i, 3),(4 i, 2),(6 i, 0): i \geq 0\}$. Then it is easily seen that $\omega(\mathcal{V}(P))=\chi(\mathcal{V}(P))=4$.

Finally, consider the following Ramsey-type conjecture, whose solution would seem to be a helpful first step in proving Conjecture 1 .

Conjecture 2. For all integers $k, \ell \geq 2$ there is an $n=n(k, \ell)$ such that every set $P$ of at least $n$ points in the plane contains $\ell$ collinear points or $k$ pairwise visible points (that is, $\omega(\mathcal{V}(P)) \geq k)$.

Note that $n(k, \ell)>(\ell-1)^{\log _{2}(k-1)}$ since the projection of the $d$-dimensional $(\ell-1) \times(\ell-1) \times \cdots \times(\ell-1)$ integer lattice has no set of $\ell$ collinear points and no $k$ pairwise visible points for $k=2^{d}+1$.

If we ask for $k$ points in general position rather than $k$ pairwise visible points, then the solution is straightforward (see [2]). It is easily proved that for all $k, \ell \geq 2$ every set of at least $\frac{1}{2}(\ell-3)(k-1)(k-2)+k+1$ points in the plane contains $\ell$ collinear points or $k$ points in general position.

\section{Acknowledgements}

Thanks to Ricardo Strausz for stimulating discussions.

\section{References}

1. James Abello and Krishna Kumar. Visibility graphs and oriented matroids. Discrete Comput. Geom., 28(4):449-465, 2002.

2. Peter Brass. On point sets without $k$ collinear points. In Discrete Geometry, vol. 253 of Monographs and Textbooks in Pure and Applied Mathematics, pp. 185-192. Dekker, New York, 2003.

3. Collette Coullard and Anna Lubiw. Distance visibility graphs. Internat. J. Comput. Geom. Appl., 2(4):349362, 1992.

4. Mike Develin, Stephen Hartke, and David Petrie Moulton. A general notion of visibility graphs. Discrete Comput. Geom., 29(4):511-524, 2003.

5. Vida Dujmović, David Eppstein, Matthew Suderman, and David R. Wood. Really straight drawings, I: Planar graphs. Submitted, 2005. 
6. Alexandr V. Kostochka and Jan Kratochvíl. Covering and coloring polygon-circle graphs. Discrete Math., 163(1-3):299-305, 1997.

7. Alexandr V. Kostochka and Jaroslav Nešetríl. Colouring relatives of intervals on the plane. II. Intervals and rays in two directions. European J. Combin., 23(1):37-41, 2002.

8. Michael Larsen, James Propp, and Daniel Ullman. The fractional chromatic number of Mycielski's graphs. J. Graph Theory, 19(3):411-416, 1995.

9. Sean McGuinness. Colouring arcwise connected sets in the plane. II. Graphs Combin., 17(1):135-148, 2001.

10. Jerzy Mycielski. Sur le coloriage des graphs. Colloq. Math., 3:161-162, 1955.

11. Edward R. Scheinerman and Daniel H. Ullman. Fractional Graph Theory. Wiley, New York, 1997.

Received September 16, 2004, and in revised form March 22, 2005. Online publication July 22, 2005. 